UDC 621.391

\title{
ANALYSIS OF CORRECTIVE PROPERTIES OF ULTRA-LONG LDPC CODES
}

\author{
Leonid O. Uryvskyi, Serhii O. Osypchuk \\ The Research Institute of Telecommunications, NTUU “KPI”, Kyiv, Ukraine
}

The ultra-long LPDC codes are examined. The errors correction by ultra-long LDPC codes is analyzed. The error-correcting capability research method for ultra-long LDPC codes is described in details. The main results in table and graph representations are shown. The comparison of ultra-long LDPC codes and BCH codes is performed by using Plotkin and VarshamovGilbert limits approximation criterion.

\section{Introduction}

Low density parity check codes (LDPC) are linear block codes. The LDPC codes check matrices have a small value of ones in its rows and columns comparably to value of zeros in these rows and columns. LDPC codes were proposed by R.Gallager in 1960 [1].

Ultra-long LDPC codes are used in state-of-the-art telecommunication systems standards. For example, LDPC codes are utilized in DVB-S2 standard (Digital Video Broadcasting Satellite - Second Generation) [2]. Together with this, the ultra-long LDPC codes bit errors equalizability is not described in DVB-S2 standard explicitly. Therefore, the significant scientific and practical task is the error-correcting capability detection for ultra-long LDPC codes. Consequently the next task is overlong LPDC codes positions definition among known block codes by comparison of their characteristics. For instance, one of the best block codes with code length more than 1000 bits are named Bose-ChaudhuriHocquenghem $(\mathrm{BCH})$. Their properties are described in [3].

The correction properties of standardized ultra-long LDPC codes are analyzed in current research work. This type of overlong LDPC codes is used in up-to-date standard DVB-S2 [2]. The ultra-long LDPC codes are researched by the instrumentality of model in standardized software MatLab [4]. Overlong LDPC codes have code length 64800 bits and have code rates in the following range: $R=0,25 \ldots 0,9$.

The relevance of work is overlong LDPC codes error correcting capabilities definition. The examined LDPC codes are used in modern telecommunications. Moreover, the overlong LDPC codes position assessment in scope of known block codes is actuality as well. This can be estimated by comparison of overlong LDPC characteristics and other known block codes.

\section{Statement of the problem}

The objective of this work is to research the ultralong LDPC codes potential error-correcting capability. It is assumed that code words are transmitted through channel in telecommunication system into decoder of receiver.

The initial conditions are:

- Code rate of $R=0,25 \ldots 0,9$ according to adopted standard DVB-S2;

- Check matrix $H$ for each LDPC code rate value;

- Fixed code word length is $n=64800$ bits.

The following tasks were defined to reach needed target:

- Encode initial information bits by using LDPC coding in MatLab software. Manually produce error bits in codeword that is imitation of noise influence. Then it will be possible to perform numerical analysis on distorted code words with definite errors count that come into LDPC decoder;

- Execute the error correction capability analysis based on statistical and numerical results. It should be performed on basis of LDPC codes with code rates of $R=0,25 \ldots 0,9$ and various errors densities in code words;

- Detect the conditions conformity degree for achievement the Plotkin and Varshamov-Gilbert limits by overlong LDPC codes [5];

- Perform the characteristics comparison of known block codes with long code length ( $n>1000)$ and ultralong LDPC codes with code length $n>64800$.

\section{Fixed code length codes error-correcting capability research}

The researched parameters for assessment of LDPC codes error-correcting capabilities with fixed code 
length 64800 bits and code rates from scope of $R=0,25 \ldots 0,9$ are the next:

- Errors count in received code word at the decoder input;

- Produced errors density in code word;

- Hard decision method iterations count for decoding the LDPC code words [1].

Performed numerical experiment is based on code words distortions imitation by inserting the certain bit errors into received code words. These manually produced errors are generated with some definite frequency ratio. The iterations number for hard decision decoding is one of the parameters, that are researched and it is the initial parameter in LDPC model simultaneously. The real LDPC check matrices dimensions are enough big, and in the issue it is required to perform a huge computations number for direct decoding methods. For example, the matrices with sizes $64800 \times R \cdot 64800$ bits are used in current work. The iterative decoding methods are used due to high complexity of direct methods. The iterative hard decision method is one of such decoding methods.

The hard decision method is proposed by Robert Gallager [1] as one of iterative methods for decoding. The hard decision method is based on iterative inverting bits ("0" to " 1 " or "1" to "0") in code word. So, firstly, the corresponding code word bit positions and rows of check matrix are multiplied. Secondly, the XOR operation is used for multiplied values of bits from code word and check matrix rows on the same positions. Thirdly, given values of XOR operations are checked on satisfying the parity condition. Fourthly, the bits inversion in code word is performed if parity condition is not satisfied. This operation is executed in code word only for bits that have maximum sum of " 1 " on exactly defined positions in non-satisfied rows.

Assume the code rate $R$ is known. Assume the information sequence is transmitted and has the length of $k=R \cdot n=R \cdot 64800$ bits. Assume the check matrix $H$ is given and has the dimensions of $64800 \times R \cdot 64800$ bits. Then received code word $w$ has the length of $n=k / R$ bits. The hard decision decoding algorithm is described shortly below:

1) The result of XOR operation is calculated for code word multiplied bit positions and corresponding check matrix rows bit positions. The vector of values is received. The vector length is equal to number of rows in check matrix. Then the parity check is executed: if the result of XOR operation is equal to " 0 " for values in vector, it is on assumption that parity check satisfied, else if value is " 1 " - parity check is not satisfied.

2) Assume the temporary matrix $H^{\prime}$ is the matrix that includes the results of multiplied code word bit positions and corresponding bit positions in rows of check matrix $H$. The sums of " 1 " for each column in matrix $H^{\prime}$ are calculated only for rows that did not pass parity check.

3) The definite positions in code word $w$ are chosen to satisfy the following condition: the sum of " 1 " on this position for rows in matrix $H^{\prime}$ is maximal comparably to other sum values of " 1 " in matrix $H$ ' rows that did not pass parity check. Thereafter the inversion for chosen positions is performed on the opposite bit values.

4) Steps 1, 2, 3 are performed for each decoding iteration. This is executed until one of two conditions is reached: 1) all parity checks are passed; 2) the compulsory stoppage condition is achieved. The compulsory stoppage condition is brought to prevent the infinite looping possibility during decoding procedure.

The initial parameter for numerical experiment is the code rate $R$. This is due to overlong LDPC codes research is performed based on model in standardized software MatLab. The research algorithm is described in details below:

1) The information sequence with length $k=R \cdot n=R \cdot 64800$ is generated.

2) LDPC encoder formed the code word $w$ with the length of $n=k / R=64800$ bits based on known code rate $R$ and check matrix $H$.

3) Bit errors $N_{e r r}$ are inculcated into code word $w$ intentionally. Assume code word with errors is named $w^{\prime}$.

4) Code word $w^{\prime}$ with bit errors $N_{\text {err }}$ came into LDPC decoder. LDPC decoder has pre-defined possible iterations limit to execute decoding procedure by hard decision method. If all parity checks satisfied before maximum iterations limit was reached, then decoder stopped the decoding procedure [1]. In this case it is supposed that information sequence is decoded correctly. In other case if iterations limit is reached, it is supposed that information sequence is not decoded properly and has bit errors. The errors number can be unknown in practice; however, it is possible to assess corrected bits count in received information sequence due to performed numerical experiments with known manually inculcated errors in received code word.

5) The difference between transmitted and decoded bit sequences is detected. 

fig. 1.

The numerical experiment algorithm is shown on

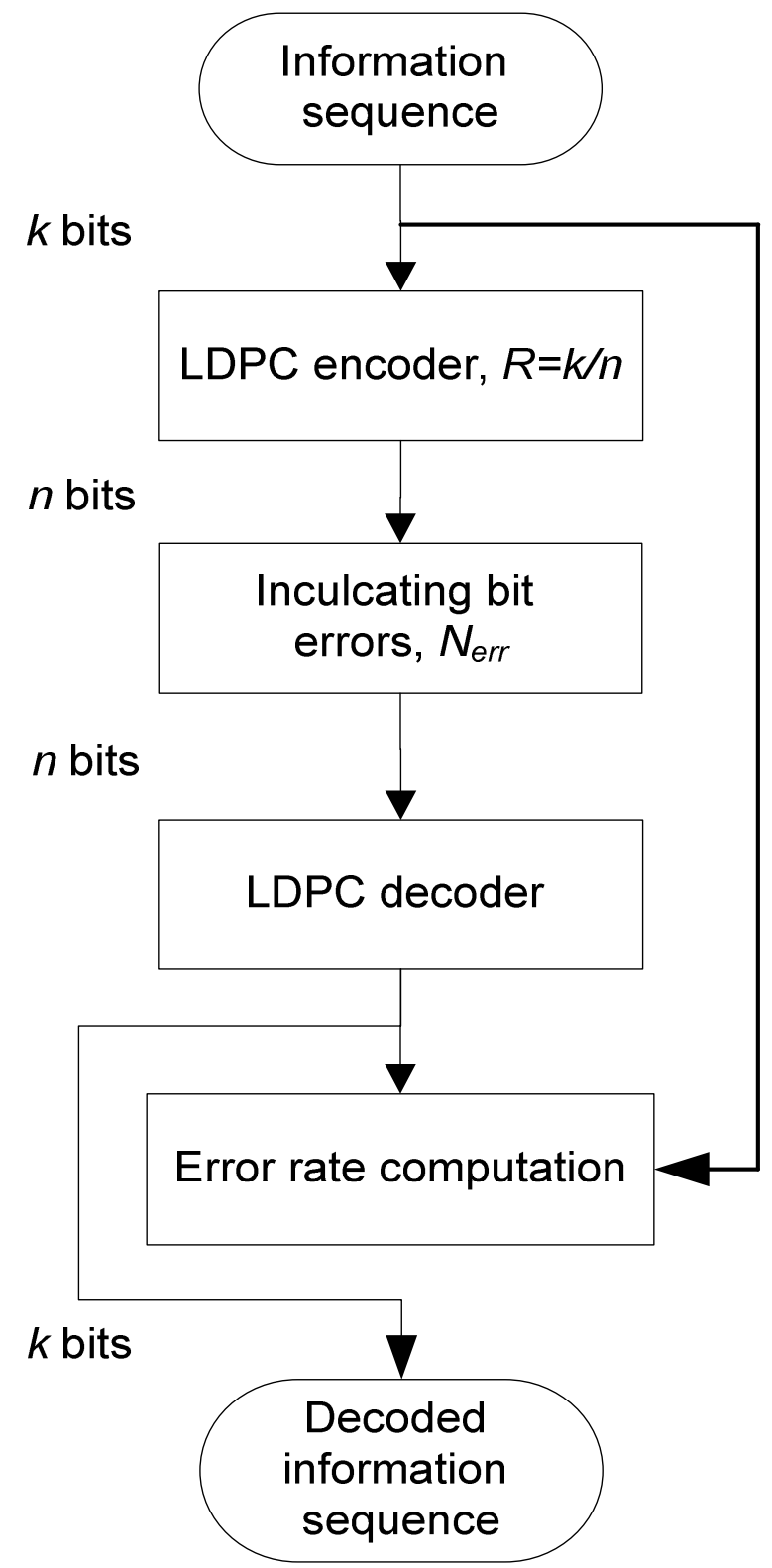

Fig. 1. The numerical experiment algorithm

The dependency of corrected errors count $C_{e r r}$ from inculcated errors $N_{e r r}$ in code word with code rate $R=1 / 2$ and iterations number for decoding 10,30 and 50 is presented on fig. 2 according to algorithm fig. 1. It is important to highlight that fig. $2-5$ are plotted only for overlong LDPC code with code rate $R=1 / 2$. The $1000,2000, \ldots, 6000$ error bits are inculcated into received code word on the first experiment phase. These errors are evenly scattered through code word. Then the corrected errors numbers are detected based on comparison of transmitted information sequence and decoded information sequence. As shown on fig. 2, if
$C_{e r r}=N_{e r r}$ then information is decoded correctly. In case $N_{e r r}>C_{e r r}>0$ the information is partially restored. If $C_{e r r}<0$ then information sequence is not decoded correctly. In addition, if $C_{\text {err }}<0$, then the additional errors are brought up into information sequence during decoding process. On fig. 2: dashed-dot line decoding iterations count is 10 ; solid and dashed lines iterations counts are 30 and 50 respectively. As shown on fig. 2, the lines for 30 and 50 iterations are almost coincidental.

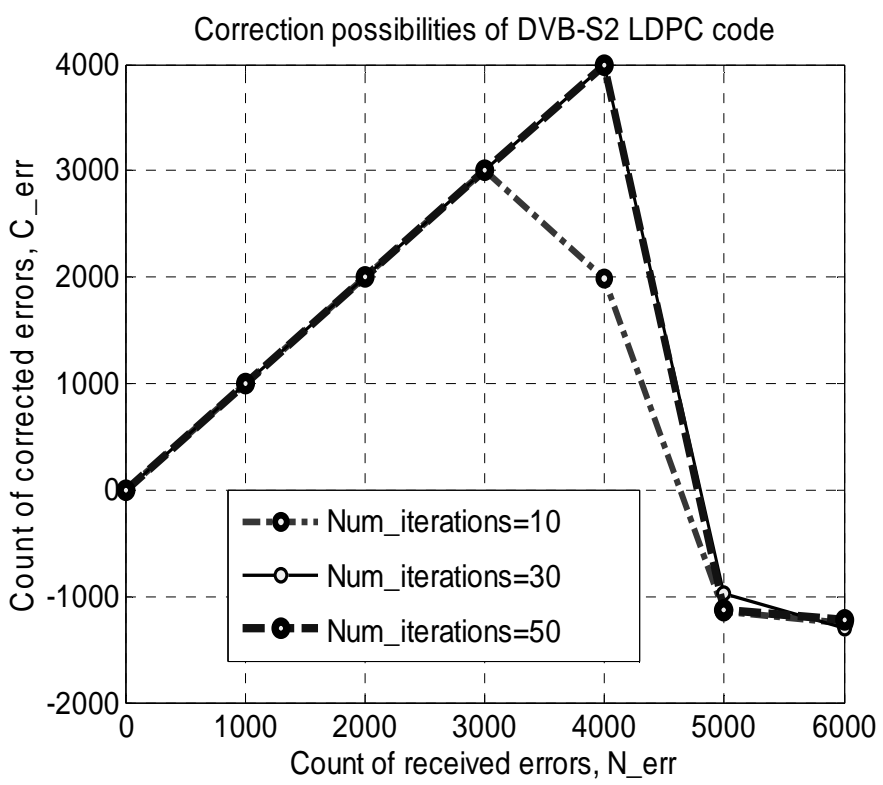

Fig. 2. The dependency of corrected errors count $C_{e r r}$ from inculcated errors count $N_{\text {err }}$

Numerical experiments shown that LDPC decoder significantly better decoded scatter bit errors comparably to block errors in the same code with the same iterations number for decoding.

The iterations count analysis shown that increasing the iterations number from 50 up to 100 practically didn't improve the decoding results. With a view to rational computing resources consuming it is reasonable to use $10 \ldots 50$ iterations for decoding. Therefore in computational experiments of current work the iterations count 30, 40 and 50 are used. These iterations are chosen by the optimum computational resources usage and received information bit integrity criteria [3].

As shown on fig. 2, LDPC decoder corrected bit errors in the range of $4000 \ldots 5000$ bits. Code word length is 64800 bits and code rate is $R=1 / 2$. The overlong LDPC code error correcting capability results are presented on fig. 3 for the case $4000,4100, \ldots, 5000$ bits are manually inculcated into received code word. 


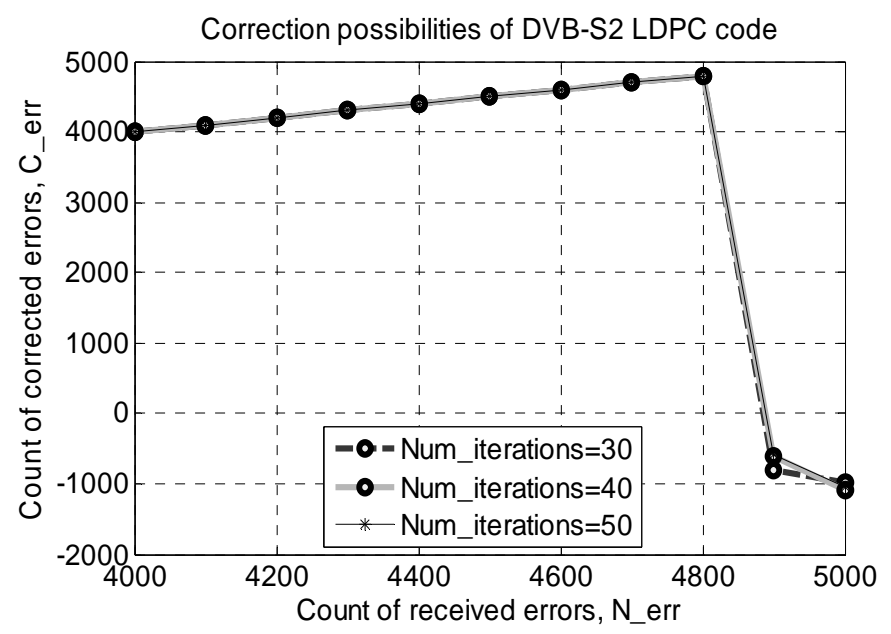

Fig. 3. The dependency of corrected errors count $C_{e r r}$ from inculcated errors count $N_{e r r}$ for $N_{e r r}=4000,4100, \ldots, 5000$

As shown on fig. 3, LDPC decoder corrected bit errors in the range of $4800 \ldots 4900$ bits. The overlong LDPC code error correcting capability results are presented on fig. 4 for the case of $4800,4810, \ldots, 4900$ bits are manually inculcated into received code word.

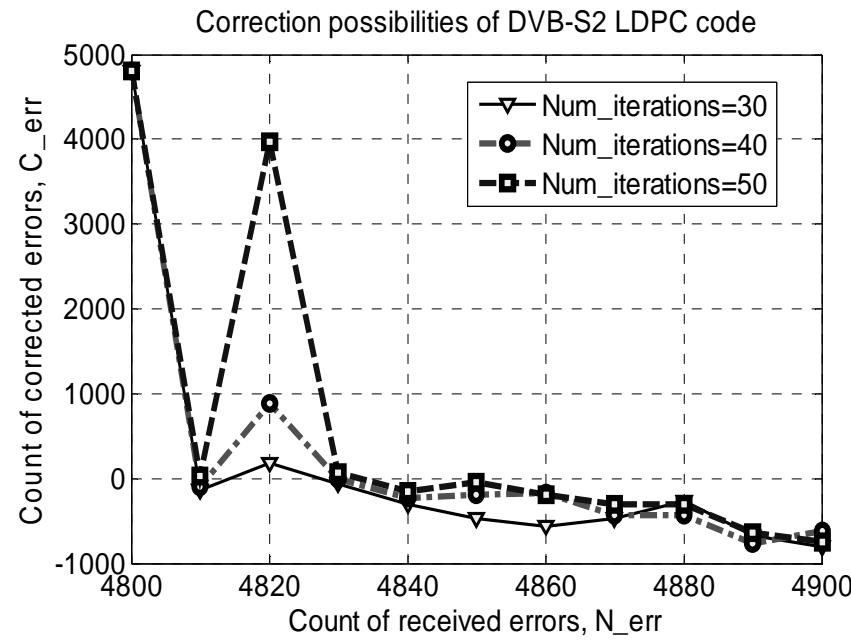

Fig. 4. The dependency of corrected errors count $C_{e r r}$ from inculcated errors count $N_{e r r}$ for $N_{e r r}=4800,4810, \ldots, 4900$

Thereby, on the fig. 4 is shown, that LDPC code corrected between $4800 \ldots 4810$ bit errors in received code words. The error correcting capability analysis in the range of $4800 \ldots 4810$ bit errors is shown on fig. 5 .

As shown on fig. 5, the number of corrected errors grew very insignificantly in parallel with iterations number. Overlong LDPC corrected 4800 errors by 30 iterations, 4803 errors by 40 iterations, 4804 by 50 iterations. Computer time for decoding grew linearly with the iterations number. Therefore, 30 iterations are enough for decoding procedure and saving computational time with similar error correction capability. It is significantly to note, that errors scattering through bits in code word positively affected the corrected errors number for the same other LDPC parameters. The best error correcting capability at level 5180 errors achieved in parallel of scattering errors through code word with $R=1 / 2$.

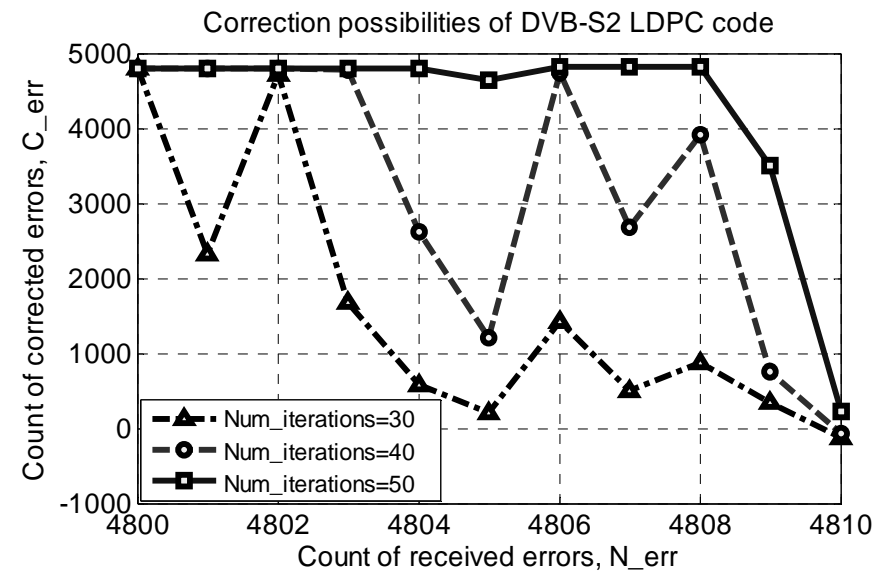

Fig. 5. The dependency of corrected errors count $C_{e r r}$ from inculcated errors count $N_{e r r}$ for $N_{e r r}=4800,4801, \ldots, 4810$

The ultra-long LDPC codes error correcting capability results with different code rates and 50 iterations for decoding are shown in table 1.

Table 1. LDPC code error correcting capability results. Code word length is $n=64800,50$ decoding iterations, scattered error bits through code word

\begin{tabular}{|c|c|c|c|}
\hline $\begin{array}{c}\text { Code rate, } \\
R=k / n\end{array}$ & $\begin{array}{c}\text { Corrected errors } \\
\text { in codeword, } t\end{array}$ & $\begin{array}{c}\text { Code } \\
\text { distance, } \\
d=2 \cdot t+1\end{array}$ & $\frac{d}{2 n}$ \\
\hline $1 / 4$ & 11270 & 22541 & 0.174 \\
\hline $1 / 3$ & 8760 & 17521 & 0.135 \\
\hline $2 / 5$ & 6200 & 12401 & 0.096 \\
\hline $1 / 2$ & 5180 & 10361 & 0.08 \\
\hline $3 / 5$ & 2945 & 5891 & 0.045 \\
\hline $2 / 3$ & 2810 & 5621 & 0.043 \\
\hline $3 / 4$ & 2060 & 4121 & 0.032 \\
\hline $4 / 5$ & 1430 & 2861 & 0.022 \\
\hline $5 / 6$ & 990 & 1981 & 0.015 \\
\hline $8 / 9$ & 610 & 1221 & 0.0094 \\
\hline $9 / 10$ & 540 & 1081 & 0.0083 \\
\hline
\end{tabular}

\section{Ultra-long LDPC codes and other known block codes characteristics comparison}

The Plotkin and Varshamov-Gilbert limits define the conditions for existence the code with some specified error correction capabilities [5]. The Plotkin limit is 
defined by conditions: if code word length is $n \geq 2 d-1$ bits, then check bits number $r=n-k$ for minimal code distance $d$ is equal at least $2 d-2-\log _{2} d$. Corollary,

$$
R \leq 1-\frac{2 d-d-\log _{2} d}{n}
$$

The $2^{\text {nd }}$ form of Plotkin limit is:

$$
k \leq n-\left(2 d-2-\log _{2} n\right)
$$

The Plotkin limit is used for codes with large code word length $n: n>>1$.

The Varshamov-Gilbert limit determines the sufficient conditions for existence the code with specified error correcting capabilities. It states that $(n, k)$-code exists with code distance value at least $d$ and the next expression will be satisfied:

$$
\sum_{i=0}^{d-2} C_{n-1}^{i} \geq 2^{n-k}
$$

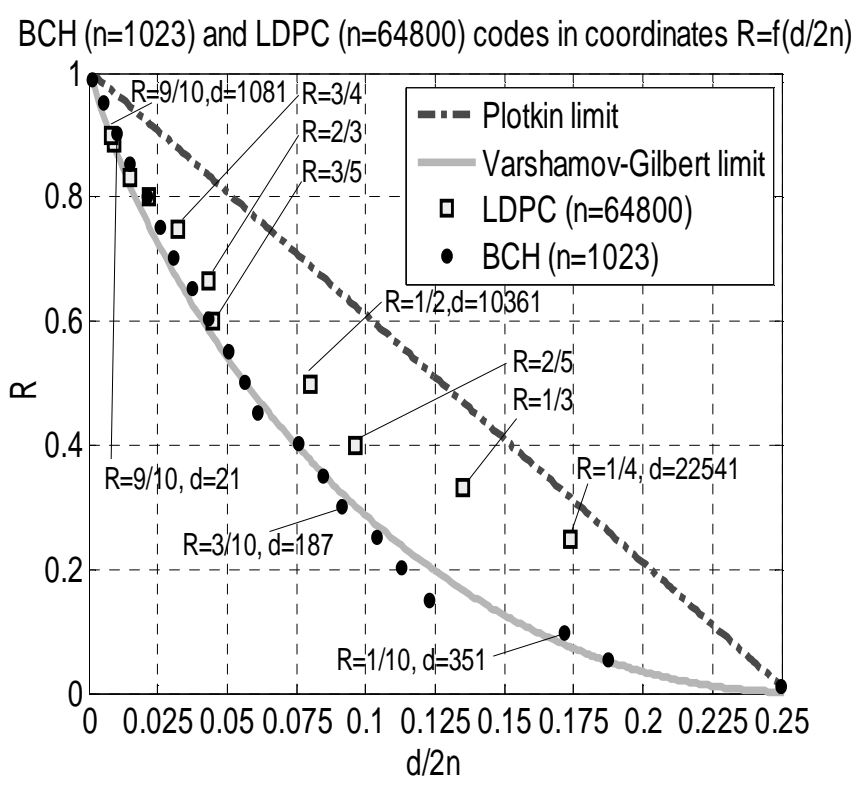

Fig. 6. $\mathrm{LDPC}$ and $\mathrm{BCH}$ codes in coordinates $R=f(d / 2 n)$

Thereby, Plotkin and Varshamov-Gilbert criteria allow bring into comparison different block codes in the same coordinates $R=f(d / 2 n)$ for assessment the error correction capabilities of compared codes.

One of the best block codes by criterion of Varshamov-Gilbert are $\mathrm{BCH}$ codes with big code length $(n>1000)$ [3]. The parameters of some BCH codes with code length $n=1023$ are presented in table 2 .

Table 2. Some known parameters of BCH codes with code length $n=1023$

\begin{tabular}{|l|l|l|c|c|}
\hline$k$ & $t$ & $d$ & $R=k / n$ & $d / 2 n$ \\
\hline 1013 & 1 & 3 & 0.990225 & 0.001466 \\
\hline 973 & 5 & 11 & 0.951124 & 0.005376 \\
\hline 923 & 10 & 21 & 0.902248 & 0.010264 \\
\hline
\end{tabular}

\begin{tabular}{|l|l|l|l|l|}
\hline 873 & 15 & 31 & 0.853372 & 0.015152 \\
\hline 818 & 21 & 43 & 0.799609 & 0.021017 \\
\hline 768 & 26 & 53 & 0.750733 & 0.025904 \\
\hline 718 & 31 & 63 & 0.701857 & 0.030792 \\
\hline 668 & 38 & 77 & 0.652981 & 0.037634 \\
\hline 618 & 44 & 89 & 0.604106 & 0.0435 \\
\hline 563 & 51 & 103 & 0.550342 & 0.050342 \\
\hline 513 & 57 & 115 & 0.501466 & 0.056207 \\
\hline 463 & 62 & 125 & 0.45259 & 0.061095 \\
\hline 413 & 77 & 155 & 0.403715 & 0.075758 \\
\hline 358 & 86 & 173 & 0.349951 & 0.084555 \\
\hline 308 & 93 & 187 & 0.301075 & 0.091398 \\
\hline 258 & 106 & 213 & 0.252199 & 0.104106 \\
\hline 208 & 115 & 231 & 0.203324 & 0.112903 \\
\hline 153 & 125 & 251 & 0.14956 & 0.122678 \\
\hline 101 & 175 & 351 & 0.098729 & 0.171554 \\
\hline 56 & 191 & 383 & 0.054741 & 0.187195 \\
\hline 11 & 255 & 511 & 0.010753 & 0.249756 \\
\hline
\end{tabular}

The Plotkin and Varshamov-Gilbert limits are plotted in coordinates $R=f(d / 2 n)$ for large code length values $(n>1000)$ on fig. 6 . The overlong LDPC codes and $\mathrm{BCH}$ codes points are plotted in fig. 6 from table 1,2 as well. As opposed to Plotkin limit, the Varshamov-Gilbert limit means, that code with some code rate, standing on this limit or above it, always exists. As shown on fig. 6, ultra-long LDPC with code length 64800 bits satisfies to this statement and stays above Varshamov-Gilbert limit. This fact characterizes the overlong LDPC code with code length 64800 bits as the best realizable code so far as overlong LDPC lies above Varshamov-Gilbert limit and below Plotkin limit. On Fig. 6 it is shown, that ultra-long LDPC codes have significantly better error correction capability comparably BCH codes. First of all, it happens because LDPC has much longer code length than $\mathrm{BCH}$.

It is possible to compare LDCP and $\mathrm{BCH}$ by relative number of corrected errors per code word criterion. So long as overlong LDPC and BCH codes have different code words length, 64800 and 1023 accordingly, then it make sense, in the first place, to choose two codes with the same code rate. For example, let's consider code rate $R=1 / 2$. Regarding tables 1 and 2 , the corrected errors number for LDPC is equal to 5180 , for $\mathrm{BCH}$ code it is 57 errors. If consider the corrected errors relation through code words of LDPC and $\mathrm{BCH}$ with the same code rate, then it is possible to talk about relative corrected errors number. For BCH code this relative corrected errors value is

$m_{\text {err }_{-} B C H}=\frac{t}{n}=\frac{57}{1023}=0.0557$, for LDPC it is

$m_{\text {err } \_ \text {LDPC }}=\frac{t}{n}=\frac{5180}{64800}=0.0799$. The relative ultra- 
long LDPC codes efficiency gain comparably to $\mathrm{BCH}$ is $\frac{m_{\text {err_ } L D P C}}{m_{\text {err_ } B C H}}=\frac{0.0557}{0.0779}=1.43$ on the code rate of $R=1 / 2$. The relative values of corrected bits per code word with pre-defined code rates scope for LDPC and $\mathrm{BCH}$ are shown in table 3.

Table 3. The relative efficiency gain of LDPC codes comparably to $\mathrm{BCH}$ codes by relative bit errors correction through code word criterion

\begin{tabular}{|c|c|c|c|c|c|}
\hline & LDPC & & BCH & & \multirow[b]{2}{*}{$(t / n)_{L D P C}$} \\
\hline & 64800 & & 1023 & & \\
\hline$R$ & $t$ & $t / n$ & $t$ & $t / n$ & $(t / n)_{B C H}$ \\
\hline 0.25 & 11270 & 0.173919753 & 106 & 0.103616813 & \begin{tabular}{l|l|} 
& 1.68
\end{tabular} \\
\hline 0.33 & 8760 & 0.135185185 & 86 & 0.084066471 & 1.61 \\
\hline 0.40 & 6200 & 0.095679012 & 77 & 0.075268817 & 1.27 \\
\hline 0.50 & 5180 & 0.079938272 & 57 & 0.055718475 & 1.43 \\
\hline 0.60 & 2945 & 0.045447531 & 44 & 0.043010753 & 1.06 \\
\hline 0.67 & 2810 & 0.043364198 & 38 & 0.03714565 & 1.17 \\
\hline 0.75 & 2060 & 0.031790123 & 26 & 0.025415445 & 1.25 \\
\hline 0.80 & 1430 & 0.022067901 & 21 & 0.020527859 & 1.08 \\
\hline 0.83 & 990 & 0.015277778 & 15 & 0.014662757 & 1.04 \\
\hline 0.89 & 610 & 0.00941358 & 11 & 0.010752688 & 0.88 \\
\hline 0.90 & 540 & 0.008333333 & 10 & 0.009775171 & 0.85 \\
\hline
\end{tabular}

The plot representation of dependency $\frac{(t / n)_{L D P C}}{(t / n)_{B C H}}=f(R)$ is shown on fig. 7.

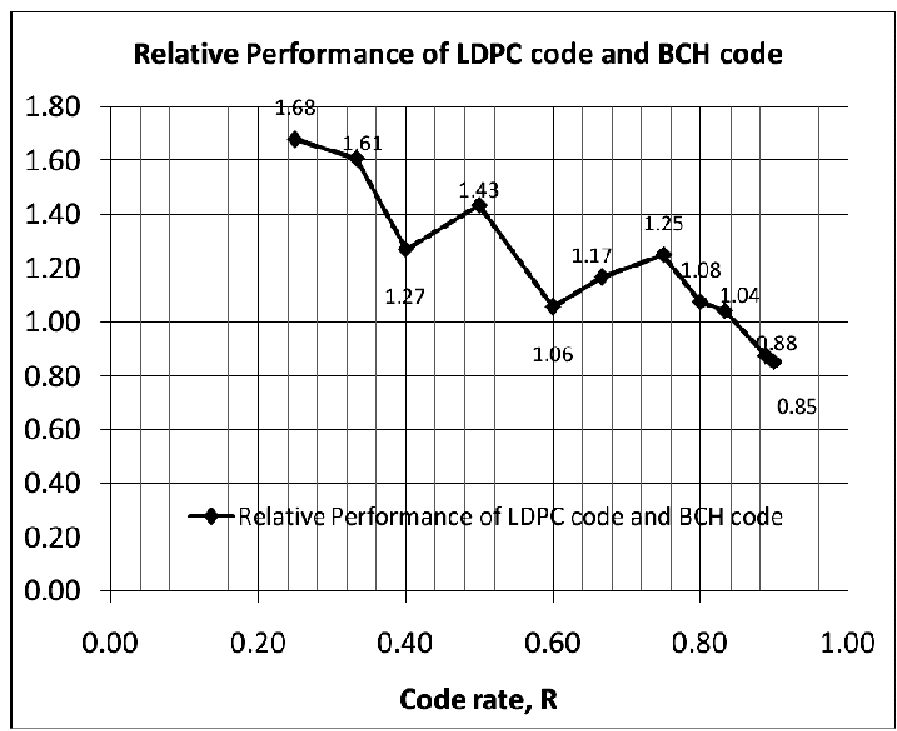

Fig. 7. The relative performance of $\mathrm{LDPC}$ and $\mathrm{BCH}$ codes: $\frac{(t / n)_{L D P C}}{(t / n)_{B C H}}=f(R)$

\section{Conclusion}

The possibility analysis to correct bit errors by ultralong LDPC codes with the code rates of $R=0,25 \ldots 0,9$ and different errors density is performed based on statistical and numerical experiments.

Based on numerical experiments it is shown, that overlong LDPC codes are better than the longest BCH codes. This conclusion is based on the criterion of achievement the potential error correcting capability bounds for block codes (Plotkin limit), and especially for code rates $R \leq 1 / 2$. At once it should be highlighted that the LDPC codes systemic deficiency is even number of code distance $d$. So LDPC codes can be classified as imperfect.

LDPC decodes error blocks considerably worse comparably to scattered bit errors through code word in case when other code parameters are constant.

The iterations number research for LDPC decoding to 100 shown, that iterations count rising practically doesn't make sense above 50 iterations so far as it doesn't improve decoding results significantly. In terms of rational computational resources using it is reasonable to use $10 \ldots 50$ iterations for decoding.

The errors density scattering through code word positively affects the corrected errors number during decoding. This is observed that the inculcated errors number in code word is the same and communication channel energy parameters are unchanged. This result indicates the practicability of interleaving schemes using in communication devices as a step that complements LDPC coding.

\section{References}

1. Low-density parity-check codes / R. Gallager. - M.: "Mir", 1966. - 144 p.

2. Digital Video Broadcasting (DVB): Second generation (DVB-S2) / ETSI EN 302307 V1.2.1 // European Standard2009. - Available:

http://www.etsi.org/deliver/etsi_en/302300_302399/302307/0 1.02.01_60/en_302307v010201p.pdf.

3. L.A. Uryvskyi, E.A. Prokopenko, A.M. Peshkin Noiseproof codes with the maximum approximation to the Shannon bound // Telecommunication Sciences. - 2011. - Volume 2, Number 1. - P.41-46.

4. Low-density parity-check codes from DVB-S.2 standard / The MathWorks, Inc. // 2012. - Available:

http://www.mathworks.com/help/comm/ref/dvbs2ldpc.html.

5. The telecommunications theory fundamentals: textbook / Under general edition of M.Yu. Ilchenko. - K. ISCIS NTUU “KPI", 2010. - 788 p.

Received in final form February 1, 2013 